\title{
Preliminary Molecular Studies of the First Report of Burkholderia pseudomallei Isolation from Soil Collected in the Amapá State, in Northern Brazil
}

V Y K Sousa ${ }^{1}$, J F O Segovia ${ }^{2}$, P O Martins Junior ${ }^{3}$, R M Bezerra ${ }^{4}$, M C A Gonçalves ${ }^{3}$, S W M Pereira ${ }^{4}$, D C Vallim ${ }^{5}$, A P D Carvalho-Assef ${ }^{6}$, E Hofer $^{5} \&$ L I B Kanzaki ${ }^{3}$

${ }^{1}$ Laboratory of Biomolecular Technology, Federal University of Pará, Pará, Brazil

${ }^{2}$ Brazilian Agricultural Research Corporation, Amapá, Brazil

${ }^{3}$ Laboratory of Bioprospection, University of Brasilia, DF, Brazil

${ }^{4}$ Laboratory of Atomic Absorption and Bioprospection, Federal University of Amapá, Amapá, Brazil

${ }^{5}$ Laboratory of Bacterial Zoonosis, Oswaldo Cruz Foundation, Rio de Janeiro, Brazil.

${ }^{6}$ Laboratory of Research in Hospital Infection, Oswaldo Cruz Foundation, RJ, Brazil.

Correspondence: L I B Kanzaki, Laboratory of Bioprospection, University of Brasilia, DF, Brazil. Tel: 55-61-3107-2846. E-mail: kanzaki@unb.br

Received: September 9, 2015 Accepted: October 9, 2015 Online Published: October 13, 2015

doi:10.5539/ijb.v8n1p9 URL: http://dx.doi.org/10.5539/ijb.v8n1p9

\begin{abstract}
The wide spectra of colonizing microorganism, likewise Burkholderia pseudomallei, has been found in different habitats, and presenting distinct activity, as exerting physiological functions among plants, or as a pathogen for man, animals and also as a phytopathogen. A common disease of men and animals caused by B. pseudomallei, melioidosis, is a severe morbidity that usually culminates in the host death. Soil samples from different areas in the Amapá state, in northern Brazil, were screened for environmental microorganisms to assess potential antimicrobial activity aiming at biotechnological applications. Among the prospected microorganisms, $B$. pseudomalle $i$ was isolated from high humidity soils, mangrove, which is rich in organic materials, produced by the diversified local flora and fauna. The isolated B. pseudomallei was identified by its biochemical profile and growth characteristics. Molecular confirmation of $B$. pseudomallei phenotypic identification was achieved by PCR amplification of the $16 \mathrm{~S}$ ribosomal DNA. The sequencing of amplified products confirmed that the Amapá sample, and two other isolates from human infections in Ceará state, northeast Brazil, were B. pseudomallei, and sequence alignement to the same specie, MSHR146 strain from Australia, and clone YN01 from uncultured Burkholderia sp., deposited in the GenBank, exhibited close phylogenetic relationship among them. Until now, there is no report of $B$. pseudomallei related disease among human and animal populations in the Amapá state, despite the finding of $B$. pseudomallei in it, in an area of water buffalo ranching and flowing small rivers utilized by human populations.
\end{abstract}

Keywords: Burkholderia pseudomallei, soil, Amapa state, Northern Brazil, gene sequences

\section{Introduction}

B. pseudomallei is a very versatile facultative Gram negative intracellular bacterium, capable to cause diseases in humans, animals and also plants (Lee et al., 2010). Melioidosis, a severe human disease caused by $B$. pseudomallei, presents a varied clinical expression and frequently culminate in the patient death, in more than $40 \%$ of the infected subjects (Currie, 2015; Hoffmaster et al., 2013). The pathogenicity of B. pseudomallei is of great concern among health authorities, so the laboratory diagnosis and studies of this organism must be carried out under the biosafety level 3, according to the Centers for Disease Control and Prevention (Emery et al., 2014). The complex genome of $B$. pseudomallei comprises 2 circular chromosomes in a total size of $7.2 \mathrm{MB}$. Genetic variations due to the high recombination rate, among different clades of $B$. pseudomallei, contribute to its elevated virulence (Challacombe et al., 2014). Phylogenetic analysis of different B. pseudomallei isolates, in the world, point out to the soil as their origin, as commonly reported in tropical and subtropical regions as the Southeast Asia, Australia and India (Radua et al., 2000; Nandagopal et al., 2012). In Brazil, few sporadic cases 
of B. pseudomallei detection, infection and related diseases have been reported (Miralles et al., 2004; Rolim et al., 2011). Here, it is described the first case of B. pseudomallei isolation from soil collected in the Amapá state, in northern Brazil, but unnoticed infection or related disease in man or animals etiologically linked to this microorganism is unknown and of great concern to health authorities due to disease severity and the high mortality rate.

\section{Material and Methods}

\subsection{Collection of Samples}

Soil samples were collected in 36 distinct geographical areas of the Amapá state (Sousa et al., 2013). One kilogram of soil was sampled in the field, at $15 \mathrm{~cm}$ deep, with a cleaned and disinfected sampling tube, and transferred to sterilized plastic bags which were kept in ice box and transported to the Laboratory of Bioprospection at the University of Brasilia, distant 3 hours by plane from the collected areas. The soil samples were kept under refrigeration at $4{ }^{\circ} \mathrm{C}$ in the laboratory until processing.

\subsection{Isolation and Identification of Soil Microorganisms}

An aliquot of $50 \mathrm{mg}$ of each soil sample was diluted in $9 \mathrm{~mL}$ of sterilized distilled water and spread out on Luria-Bertani agar utilizing an $1 \mu \mathrm{L}$ inoculation loop. After 24 hours incubation at $37^{\circ} \mathrm{C}$, each grown colony was transferred into $4 \mathrm{~mL}$ of Luria Bertani broth under the same conditions previously reported. The purity of the colony was confirmed by growth in the same agar. The supernatant of purified grown colonies was filtered and tested for antimicrobial activity as described in Sousa et al. (2013). Bacterial species whose supernatant showed activity against pathogenic microorganisms were identified by classical and automated methods (VITEK 2 automated identification system) utilizing the Biomerieux phenotypic identification kit. A soil sample collected in the Amapá county showed colonies that were confirmed to be B. pseudomallei, in the Laboratory of Bacterial Zoonosis, Oswaldo Cruz Institute/FIOCRUZ, Rio de Janeiro, RJ, Brazil, utilizing the methodology described in Miralles et al. (2004), as briefly described. Isolated colonies, presenting characteristics of the Burkholderia genus, sent from the Laboratory of Bioprospection (University of Brasilia), were plated onto a selective MacConKey agar and also, on nutrient agar with $5 \%$ of defibrinated sheep blood. Red colonies in MacConkey agar and non hemolytic in blood agar were subcultured in presumptive media (Pessoa \& Rugai and Kligler agar). Preliminary analyses were performed according to Hofer (Hofer, E. Gram negative non fermentative bacteria, 1985 - not published), which includes the oxidase test, action of glucose in O-F medium and motility on semisolid agar plate, besides considering the previous growth on MacConkey agar. Samples were analysed in triplicate and results confirmed by repeating twice.

\subsection{Molecular Analysis of B. pseudomallei Samples}

\subsubsection{DNA Extraction}

Twenty-four hour growth culture of one colony of B. pseudomallei isolate in $5 \mathrm{~mL}$ of Brain Heart Infusion broth was centrifuged at $12,500 \mathrm{Xg}$ for 5 minutes, and the cell sediment was washed three times in $1 \mathrm{~mL}$ of saline buffer and resuspended in $180 \mu \mathrm{L}$ of genomic digestion buffer and $20 \mu \mathrm{L}$ of proteinase K (Invitrogen, US). After incubation at $55^{\circ} \mathrm{C}$ for 15 hours, $20 \mu \mathrm{L}$ of RNAse was added and homogenized. Sequentially, $200 \mu \mathrm{L}$ of lysis genomic buffer was added to the mixture and vortexed, and $200 \mu \mathrm{L}$ of absolute ethanol was added to the homogenized solution and mixed for 5 seconds. The lysed solution was transferred to a spin column, adjusted to a tube collector, and centrifuged for 10,000Xg for 1 minute. The spin column was washed two times with 500 $\mu \mathrm{L}$ of washing buffer for 3 minutes at $15,300 \mathrm{Xg}$. DNA elution was carried out after transference of the spin column to a DNAse free eppendorf tube and adding $200 \mu \mathrm{L}$ of genomic elution buffer. After centrifugation, the eluted solution was kept at $-20{ }^{\circ} \mathrm{C}$ until utilization. DNA quality was ascertained by running the sample in $0.75 \%$ agarose gel and visualization after ethidium bromide staining.

\subsubsection{DNA Amplification by PCR}

The DNA obtained from B. pseudomallei isolated from Amapá soil, and humans strains from Ceará state, kept at the Bacterial Zoonosis Laboratory/IOC/FIOCRUZ, were amplified by the Polymerase Chain Reaction utilizing primers targeting the $16 \mathrm{~S}$ rRNA genes (Bolivar et al., 2012) (fD1- AGAGTTTGATCCTGGGTCAG/Rp1 ACGGTTACCTTGTTACGACTT). Briefly, a mix containing $25 \mu \mathrm{L}$ of ReadyMix Taq PCR reaction with $\mathrm{MgCl}_{2}$ (Sigma, St. Louis, US), $2 \mu \mathrm{L}$ of each primer $(10 \mu \mathrm{M}), 1 \mu \mathrm{L}$ of DNA and $20 \mu \mathrm{L}$ of MilliQ water, were submitted to 30 cycles of $94{ }^{\circ} \mathrm{C} / 1 \mathrm{~min} ., 55^{\circ} \mathrm{C} / 2 \mathrm{~min}$. and $72{ }^{\circ} \mathrm{C} / 3 \mathrm{~min}$. Gene amplification was confirmed by running the obtained products in $0.75 \%$ agarose gel and ethidium bromide staining. 


\subsubsection{Sequencing of Amplified Products}

PCR amplified products were purified using Millipore plate MSNU030 (Millipore SAS, Molsheim, France), and Sanger-sequenced in a MJ Research PTC-225 Peltier Thermal Cycler (Bio-Rad) using an ABI PRISM BigDyeTM Terminator Cycle Sequencing Kits with AmpliTaq DNA polymerase (FS enzyme), following the protocols supplied by the manufacturer. Single-pass sequencing was performed on each template using the same primers employed initially to amplify the $16 \mathrm{~S}$ rRNA genes. The fluorescent-labeled fragments were purified from the unincorporated terminators with BigDye ${ }^{\circ} X$ Terminator $^{\mathrm{TM}}$ purification protocol. The samples were resuspended in distilled water and subjected to electrophoresis in an ABI 3730xl sequencer, 96 capillary type (Applied Biosystems, Foster City, CA) at the Macrogen sequencing facility (Macrogen Inc., Seoul, Korea).

\subsubsection{Nucleotide Sequence Analysis of B. pseudomallei}

Obtained sequences were analyzed utilizing the on line suite of programs, Blast, available at the NCBI Blast homepage (http://blast.ncbi.nlm.nih.gov/Blast.cgi?CMD=Web\&PAGE_TYPE=BlastHome), by aligning the $B$. pseudomallei sequences from samples of the states of Amapá and Ceará to the B. pseudomallei GenBank deposited sequences, MSHR146 strain from Australia, and clone YN01 from uncultured Burkholderia sp. from China (Challacombe et al., 2014; Pan et al., 2015).

\subsubsection{Identification of Potential Proteins Products}

Nucleotide query with sequences of amplified products of B. pseudomallei isolates of Amapá and Ceará, yielded hypothetical putative proteins by the use of a Blast program, blastx, available at http://blast.ncbi.nlm.nih.gov/ Blast.cgi?PROGRAM=blastx\&PAGE_TYPE=BlastSearch\&LINK_LOC=blasthome. All molecular analysis were repeated for confirmation.

\section{Results}

A soil sample collected from a mangrove area in the Amapá county ( $\left.02^{\circ} 03^{\prime} 18.9^{\prime \prime} \mathrm{W} 050^{\circ} 48^{\prime} 39.6^{\prime \prime}\right)$, Amapá state, yield bacterial colonies in the agar that reacted positively in the oxidase test, presented motility and oxidized glucose. Gram staining revealed colonies to be Gram negative bacilli, in single or in palisade arrangement. A bipolar-staining bacilli was ascertained by the Wayson staining. Growth on nutrient agar with $3 \%$ glycerol, exhibited exuberant colonies with dull, wrinkled, corrugated surface and radiating ridges after 96 hours at $37^{\circ} \mathrm{C}$; the bacterium was motile in semisolid agar, at $37^{\circ} \mathrm{C}$ and room temperature. Growth characteristics and biochemical profile, confirmed by the analysis carried out in classical and automated methods. The VITEK 2 automated identification system phenotypically identified the species placing them at $81.83 \%$ probability to be B. pseudomallei. Among different types of collected soil samples, from savannah areas, high forests, and inundated areas, the microorganism was isolated from high humidity soils, mangrove, which is rich in organic materials, produced by the diversified flora and fauna.

The amplification of DNA sequences extracted from isolates of the Amapá soil and strains isolates from Ceará state, showed bands at exactly the same position in the agarose gel after electrophoresis (Figure 1). The gene sequences obtained from the amplified products of $16 \mathrm{~S}$ Ribosomal DNA of the isolated B. pseudomallei from Amapá and Ceará states are shown in the complementary information. Alignment of gene sequences from amplified products of Amapá and Ceará isolates showed close phylogenetic proximity among them, as also when aligning to two other gene sequences deposited at the GenBank (Table 1). Also, the simulated translated proteins of nucleotide sequences obtained from Amapá and Ceará samples yield the hypothetical putative proteins organized in the complementary information.

Table 1. Alignment percentage of B. pseudomallei genomic sequences of amplified products obtained from Amapá and Ceará state isolates 1 and 2, as also from deposited sequences in the GenBank

\begin{tabular}{lc}
\hline \multicolumn{1}{c}{ Aligned sequences } & Nucleic Acid Homology (\%) \\
\hline AMAPA X CEARA1 & 80 \\
AMAPA X CEARA2 & 83 \\
CEARA1 X CEARA2 & 87 \\
AMAPAX Burkholderia pseudomallei MSHR146 chromosome 2, complete sequence & 96 \\
CEARA1 X Burkholderia pseudomallei MSHR146 chromosome 1, complete sequence & 90 \\
CEARA2 X Uncultured Burkholderia sp. clone YN01 16S ribosomal RNA gene, & 90 \\
partial sequence & \\
\hline
\end{tabular}




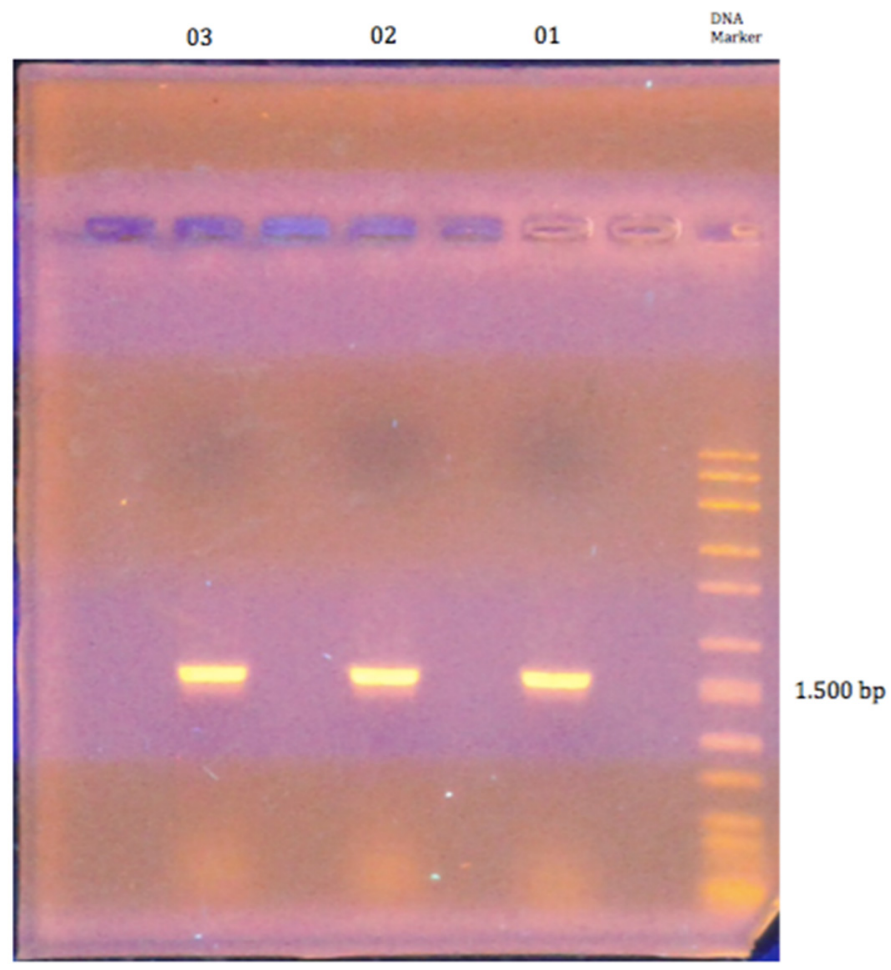

Figure 1. 16S Ribosomal DNA amplification in DNA fragments of phenotypically identified Burkholderia pseudomallei of Amapa (line 1) and Ceara state (line 2 and 3)

\section{Discussion}

Initial experiments to detect environmental bacteria in the Amapá state exhibiting antimicrobial activity against human and animal pathogenic bacteria, allowed us to isolate a highly pathogenic environmental microorganism, B. pseudomallei, which also produced metabolites presenting antimicrobial activity (Sousa et al., 2013; Iliukhin et al., 2011). Up to date, there is no report in the literature concerning human or animal infected by $B$. pseudomallei in the Amapá state, or even in the Amazon area of Brazil, although unidentified cases could have occurred.

Similarly to our findings of $B$. pseudomallei isolation from mangrove, it has been commonly reported the microorganism isolation from moist soil and water (Prakash et al., 2014; Knappik et al., 2015; Liu et al., 2015). Also, the high pluviometric index in the Amapá state (Segovia et al., 2011), as part of the Amazon region of Brazil, contributes to maintain the elevated soil humidity and natural collections of water. The Amapá county is intertwined by many rivers, exhibiting a rich fauna and flora. Cattle ranching is the main economic activity around the Amapá small town. Water buffaloes are spread out in the natural flood plains of the county and contribute to meat and milk production in the state (http://www.alice.cnptia.embrapa.br/alice/bitstream/doc/ 345690/1/IMPACTOSAMBIENTAISDABUBALINOCULTURANOSCAMPOS.pdf). Despite the isolation of $B$. pseudomallei in areas where domestic and wild animals inhabit, as also areas that communicate with rivers utilized by human populations, it is not known any notified cases of infection/disease by this microorganism.

Genetic sequences of amplified fragments of the $16 \mathrm{~S}$ ribosomal DNA of B. pseudomallei showed close phylogenetic relationship among the Amapá and Ceará isolates, as also when comparing to an Australian isolate, MSHR146 strain, and a sequence from uncultured Burkholderia sp., clone YN01, from China (Table 1). It seems that $B$. pseudomallei isolates are well conserved all around the world. This assumption is once again confirmed by the alignment of translated proteins from nucleotide sequences of amplified products of the $16 \mathrm{~S}$ ribosomal DNA of B. pseudomallei to protein sequences of the GenBank database, as shown in complementary information. The majority of the translated proteins resembles protein sequences of distinct species of the Burkholderia genus (B. mallei, B. multivorans, B. bannensis, B. pyrrocinia, B. oxyphila, B. phytofirmans, B. fungorum, B. ginsengisoli, $B$. dilworthii, B. bryophila and $B$. caledonica) (http://blast.ncbi.nlm.nih.gov/Blast.cgi? $\mathrm{CMD}=$ Web\&PAGE _TYPE=BlastHome). Even though the $16 \mathrm{~S}$ ribosomal DNA codes for the $16 \mathrm{~S}$ ribosomal RNA that contains about 1540 nucleotides, organized in the small ribosomal subunit (SSU) with 24 ribosomal 
proteins (Smith et al., 2008), it is not reported that the amplified sequences code for proteins, but gene regulation, switching sequences as introns or exons, could possibly explain the translated proteins corresponding to the mentioned sequence of $B$. pseudomallei. All proteins translated from the B. pseudomallei $16 \mathrm{~S}$ ribosomal gene sequences of the Amapá and Ceará isolates were mined from the GenBank. Gee et al. (2003) utilized the $16 \mathrm{~S}$ DNA sequence to phylogenetically compare different species of the Burkholderia genus.

\section{Conclusion}

It would be of great significance to look for coding genetic sequences and corresponding translated proteins that could be involved in the pathogenicity of $B$. pseudomallei. In conclusion, further studies of ecology and epidemiology are needed to unravel the relationship between the soil and the organism, and molecular typing methods such as those described here which greatly assisted such analysis.

\section{Acknowledgements}

This research work was funded by ELETRONORTE, Brasilia, Brazil. We thank Miss Liliane Miyuki Seki for her technical support.

\section{References}

Bolivar, I., Whiteson, K., Stadelmann, B., Baratti-Mayer, D., Gizard, Y., Mombelli, A., ... Geneva Study Group on Noma (GESNOMA). (2012). Bacterial diversity in oral samples of children in niger with acute noma, acute necrotizing gingivitis, and healthy controls. PLoS Neglected Tropical Diseases, 6(3), e1556. http://dx.doi.org/10.1371/journal.pntd.0001556.

Challacombe, J. F., Stubben, C. J., Klimko, C. P., Welkos, S. L., Kern, S. J., Bozue, J. A., ... Wolfe, D. N. (2014). Interrogation of the Burkholderia pseudomallei genome to address differential virulence among isolates. PLoS One, 9(12), e115951. http://dx.doi.org/10.1371/journal.pone.0115951.

Currie, B. J. (2015). Melioidosis: evolving concepts in epidemiology, pathogenesis, and treatment. Seminars in Respiratory and Critical Care Medicine, 36(1), 111-25. http://dx.doi.org/10.1055/s-0034-1398389.

Emery, F. D., Stabenow, J. M., \& Miller, M. A. (2014). Efficient inactivation of Burkholderia pseudomallei or Francisella tularensis in infected cells for safe removal from biosafety level 3 containment laboratories. Pathogens and Disease, 71(2), 276-81. http://dx.doi.org/10.1111/2049-632X.12138.

Gee, J. E., Sacchi, C. T., Glass, M. B., De, B. K., Weyant, R. S., Levett, P. N., ...Popovic, T. (2003). Use of 16S rRNA gene sequencing for rapid identification and differentiation of Burkholderia pseudomallei and $\mathrm{B}$. mallei. Journal of Clinical Microbiology, 41(10), 4647-54.

Hoffmaster, A. R., AuCoin, D., Baccam, P., Baggett, H. C., Baird, R., Bhengsri, S., ... Walke, H. T. (2013). Melioidosis diagnostic workshop. Emerging Infectious Disease, 21(2). http://dx.doi.org/10.3201/eid2102. 141045.

Iliukhin, V. I., Merinova, L. K., Ageeva, N. P., \& Senina, T. V. (2011). Bacteriocins and bacteriophages of pathogenic Burkholderia. Zhurnal mikrobiologii, epidemiologii, i immunobiologii, (1), 12-9.

Knappik, M., Dance, D. A, Rattanavong, S., Pierret, A., Ribolzi, O., Davong, V., ...Dittrich, S. (2015). Evaluation of molecular methods to improve the detection of Burkholderia pseudomallei in soil and water samples from Laos. Applied Environmental Microbiology, 81(11), 3722-7. http://dx.doi.org/10.1128/AEM. 04204-14.

Lee, Y. H., Chen, Y., Ouyang, X., \& Gan, Y. H. (2010). Identification of tomato plant as a novel host model for Burkholderia pseudomallei. BMC Microbiology, 10, 28. http://dx.doi.org/10.1186/1471-2180-10-28

Liu, X., Pang, L., Sim, S. H., Goh, K. T., Ravikumar, S., Win, M. S., ...Chai, L. Y. (2015). Association of melioidosis incidence with rainfall and humidity, Singapore, 2003-2012. Emerging Infectious Disease, 21(1), 159-62. http://dx.doi.org/10.3201/eid2101.140042.

Miralles, S. I, Maciel, M. C. A., Angelo, M. R. F., Gondini, M. M., Frota, L. H. F., dos Reis, C. M. F., \& Hofer, E. (2004). Burkholderia pseudomallei: a case report of a human infection in Ceará, Brazil. Revista do Instituto de Medicina Tropical de São Paulo, 46(1), 51-54. http://dx.doi.org/10.1590/S0036-46652004 000100011

Nandagopal, B., Sankar, S., Lingesan, K., Appu, K. C., Sridharan, G., \& Gopinathan, A. (2012). Application of Polymerase Chain Reaction to Detect Burkholderia pseudomallei and Brucella Species in Buffy Coat from Patients with Febrile Illness Among Rural and Peri-Urban Population. Journal of Global Infectious Disease, 4(1), 31-37. http://dx.doi.org/10.4103/0974-777X.93759. 
Pan, Y., Li, N., Mu, J., Zhou, R., Xu, Y., Cui, D., \& Wang, Y. M. (2015). Biogenic magnetic nanoparticles from Burkholderia sp. YN01 exhibiting intrinsic peroxidase-like activity and their applications. Applied Microbiology and Biotechnology, 99(2), 703-15. http://dx.doi.org/10.1007/s00253-014-5938-6.

Prakash, A., Thavaselvam, D., Kumar, A., Kumar, A., Arora, S., Tiwari, S., ... Sathyaseelan, K. (2014). Isolation, identification and characterization of Burkholderia pseudomallei from soil of coastal region of India. Springerplus, 3, 438. http://dx.doi.org/10.1186/2193-1801-3-438.

Radua, S., Ling, O. W, Srimontree, S., Lulitanond, A., Hin, W. F., Yuherman, L. S., .. Mutalib, A. R. (2000). Characterization of Burkholderia pseudomallei isolated in Thailand and Malaysia. Diagnostic Microbiology and Infectious Disease, 38(3), 141-5. http://dx.doi.org/10.1016/S0732-8893(00)00189-9

Rolim, D. B., Vilar, D. C., de Góes C. L. P, Freitas, L. B., Inglis, T. J., Nobre, R. J. L., \& Nagao-Dias, A. T. (2011). Burkholderia pseudomallei antibodies in individuals living in endemic regions in Northeastern Brazil. American Journal of Tropical Medicine and Hygiene, 84(2), 302-5. http://dx.doi.org/10.4269/ajtmh. 2011.10-0220.

Segovia, J. F. O., Oliveira, L. O., Gonçalves, M. C. A., Resck, I. S., Silva, C. A. M., Silveira, D., ...Kanzaki, L.I.B. (2011). Botanical characterization, geographical distribution and phytochemistry analysis of Manilkara huberi (Ducke) Stanhl autochtonous in Amapá State, Brazil. Vesty (National Academy of Sciences of Belarius), 2, 30-40.

Smith, T. F., Lee, J. C., Gutell, R. R., Hartman, H. (2008). The origin and evolution of the ribosome. Biology Direct, 3, 16. http://dx.doi.org/10.1186/1745-6150-3-16.

Sousa, V. Y. K., Oliveira, A. A., Segovia, J. F. O., Gonçalves, M. C. A., Bezerra, R. M., Martins-Junior, P. O. ... Hanada, R. E. (2013). Antimicrobial bioprospection among environmental bacteria in the amazon biome. Revista Biomedica, 24, 45-52.

\title{
Appendices
}

1. Genomic sequences of B. pseudomallei PCR amplified 16 S Ribossomal DNA of Amapá and Ceará States isolates.

\begin{abstract}
1. Genomic sequence of Amapá isolate of B. pseudomallei (1266 bases)
ACCCGGAACGGTTACTGTAAGTGCAAGTCGAACGGCAGCACGGGCTTCGGCCTGGTGGCGAGTGGC GAACGGGTGAGTAATACATCGGAACACCTGTAGTGGGGGATAGCCCGGCGAAAGCCGGATTAATACC GCATACGATCTGAGGATGAAAGCGGGGGACCTTCGGGCCTCGCGCTATAGTTGGCCGATGGCTGATT AGCTAGTTGGTGGGGTAAAGGCCTACCAAGGCGACGATCAGTAGCTGGTCTGAGAGGACGACCAGC CACACTGGGCTGAGACACGGCCCAGACTCCTACGGGAGGCAGCAGTGGGGAATTTGGACAATGGG CGCAAGCCTGATCCAGCAATGCCGCGTGTGTGAAGACCTTCGGGTTGTAAAGCACTTTTGTCCGGA AAGAAATCATTCTGGCTAATACCCGGAGTGGATGACGGTACCGGAAGAATAAGCACCGGCTAACTAC GTGCCAGCAGCCGCGGTAATACGTAGGGTGCGAGCGTTAATCGAATTACTGGGCGTAAAGCGTGCGC AGGCGGTTTGCTAAGACCGATGTGAAATCCCCGGGCTCAACCTGGGAACTGCATTGGTGACTGGCA GGCTAGAGTATGGCAGAGGGGGGTAGAATTCCACGTGTAGCAGTGAAATGCGTAGAGATGTGGAGG AATACCGATGGCGAAGGCAGCCCCCTGGGCCAATACTGACGCTCATGCACGAAAGCGTGGGGAGCA AACAGGATTAGATACCCTGGTAGTCCACGCCCTAAACGATGTCAACTAGTTGTTGGGGATTCATTTCC TTAGCAACGTAGCTAACGCGTGAAGTTGACCGCCTGGGGAGTACGGTCGCAAGATTAAAACTCATAG GAATTGACGGGGACCCGCACAAGCGGTGGATGATGCGGATTAATTCGATGCAACGCGAAAAACCTT ACCTACCCTTGACATGGTCGGAAGCCCGATGAGAGTGGGCTTGCTCCAAAAGAAACCGGCCCACAG GGGCTGAATGGCTATCGTCATCTCTTGGCGGAGAAAGTTTGGTTAACTCCCGCACCAGCCCAACCTT GGCCTTATGTGGTACGCAAGAGCCT
\end{abstract}

\section{Genomic sequence of Ceará isolate of $B$. pseudomallei (1000 bases)}

ACCCGCACCGAGAACACATCGCGGGACGTCCTCATTGCGGTTAGACTAGCCACTTCTGGTAAAACCC ACTCCCATGGTGTGACGGGCGGTGTGTACGAGAGCCGGGAACGTATTCACCGCGGCATGCTGATCCG CGATTACTAGCGATTCCAGCTTCATGCACTCGAGTTGCAGAGTGCAATCCGGACTACGATCGGTTTTC TGGGATTGGCTCCCCCTCGCGGGTTGGCGACCCTCTGTTCCGACCATTGTATGACGTGTGAAGCCCT ACCCATAAGGGCCATGTAGAGACTTGACGTCATCCCCACCTTCCTCCGGTTTGTCACGCGAGTTGGC TCCTTAGAGTGCTCTTGCGTAGCAACTAGCGACGAGGGTTGCGCTCGTTGCGGGACTTAACCCAACA TCTCACGACACGAGCTGACGACTGCCATGCAGCACCTGTGCGCCGGTTCTCTTTCGAGCACGCCCA ACTCTCATCGGGCTTCCGACCATGTCAAGGGAAGGTGAGGGTTTTCGCGTTGCATCAAATTAGTCCA CATCATCCGCCGCTTGTGCGGGTCCACATCAATTCCTTTGAGTTTTAATCTTGCGACCGTACTCACCG 
GGCAGTGAACTTCTCGCGTTAGCTACATGGCTAAAGAAATGCATCCGCAACAACTAGTTGACATCGA TTATGGCGTGGACTACCAGGCTATCGAATCCTGTTTGCTCAACACGCTCTCGCGCGTGAGCGTGCGT GTTGACCAACGGGATGCCATCGCCAGGAGTATACGTCACATCTCATCGCATTAACTGCTGCACATGCA ATTCTACCGCCCTCTGCCATACTCTAGACTGCCAGTCACCGATGCAGTTCGCTCGCTAGATCCAGGCG ATTTCACATCGATCGTAAGCCAACAGCTGCGCACGCTTTGCGGCCTGTTATTGCTGATAAGCGAACG CCCTCACTTCTTTCCGCGGCTGCTGAAACGCAATTAGACCGGGGCTTATTCTTCAGGAA

\section{Genomic sequence of Ceara isolate of $B$. pseudomallei (1213 bases)}

AAAGGGGAAAAGCGTCATGATCACACGCATACGTCCTCATTGCGGTTAGACTAGCCACTTCTGGTAA AACCCACTCCCATGGTGTGACGAGGCGGCGTGTACAAGACCGGGGAACGTATTCACCGCGGCATGC TGATCCGCGATTACTAGCGATTCCAGCTTCATGCACTCGAGTTGCAGAGTGCAATCCGGACTACGATC GGTTTTCTGGGATTAGCTCCCCCTCGCGGGTTGGCAACCCTCTGTTCAGACCATTGTATGACGTGTGA AGCCCTACCCATAACGCCCATGAGGACTTGACGTCCTCCCCACCTTCCTCCGGTTTGTCACCGGAATT CTCCTTAGAGTGCTCTTGCGGAGCAACTAATGACAAGGGTTGCGCTCGTTGCGGCACTTAACCCAAC ATCTCACGACACGAGCTGACGACTGCCATGCAGCACCTGTGCGCAGGATCTCTTTCGAGCACTCCCG AATCTCTACAGGATTCCGACCATGTCAAGGGTAGGTAAGGGTTTTCGCGTTGCATCGAATTAATGCAC ATCATCCACCGCTTGTGCGGGTCCTCGTAAATTCCTTTGAGCTTTAATCTTGCGACGGTACTCCCCAT GCGGACTACTTCACGCGATAGCTACATAACTAAGGAAATGTATCCGCGACAACTAGATGACATCATAT ATGGCGAGGACTACCAGGGGATCTAATCCTGTTTGCTCCCCACGCTTTCGCGCATGAACGACAGTAT GGGGCCCAGGGGGCTGCCTTCGCCCTCGGTATTCCTCCACATATCTACGCATTTCACTGCTACGCGGA GTAATTCTACCCCCCCTCTGCCATACTCTAGGCAAGCTAGACCCCCATGGGAGTTCCCAGGTTGAGA CCAGGGATTTCACGATTTGTCTGAGCAAACCGACAAGCGGACGCTTTACGCCCATTAATTCCAATTA ACGCTTAGACCCTTACGTTTACCTGGAGTTGCTGGGAACTCAATTAAGCCAGTCGGTAATTCTTCCAG AAACAACCTCCCCCCAACTGTTTTAAAAGCCAAGGATTTCTTTCCGGGAAAAAAATGCTTTAATAAC CCCAAGGCATTATTCCAAACCTTGGCCTTTTGTTGGATCAGGAATTACCCCCCTTTGGGCAAAAAG

2. Hypothetical putative proteins yielded by the use of a nucleotide query with sequences of amplified products of $B$. pseudomallei isolates of Amapá and Ceará utilizing a Blast program, blastx, available at http://blast.ncbi.nlm.nih.gov/Blast.cgi?PROGRAM=blastx\&PAGE_TYPE=BlastSearch\&LINK_LOC=blasthome.

Amapá sequence of and putative proteins

1. hypothetical protein DP49_2425 [Burkholderia pseudomallei]

2. hypothetical protein DM75_2176 [Burkholderia mallei]

3. hypothetical protein DP49_5430 [Burkholderia pseudomallei]

4. conserved hypothetical protein [Burkholderia multivorans ATCC 17616]

5. hypothetical protein Y601_3232 [Burkholderia pseudomallei MSHR640]

6. hypothetical protein DP49_5431 [Burkholderia pseudomallei]

7. conserved hypothetical protein [blood disease bacterium R229]

8. hypothetical protein [Cupriavidus basilensis]

9. sensor histidine kinase/response regulator LuxN [Cupriavidus basilensis]

Ceara 01 sequences and putative proteins

1. hypothetical protein BMULJ_05097 [Burkholderia multivorans ATCC 17616]

2. glucokinase [Ralstonia sp. UN $\bar{N}$ C404CL21Col]

3. nuclease [Burkholderia sprentiae]

4. XRE family transcriptional regulator [Burkholderia pseudomallei]

5. XRE family transcriptional regulator [Burkholderia pseudomallei]

6. dimethyl sulfoxide reductase [Burkholderia bannensis]

7. hypothetical protein HMPREF0551_2597 [Lautropia mirabilis ATCC 51599]

8. dimethyl sulfoxide reductase [Burkholderia oxyphila]

9. DNA topoisomerase III [Burkholderia pyrrocinia]

10. XRE family transcriptional regulator [Burkholderia oxyphila]

11. XRE family transcriptional regulator [Burkholderia sp. RPE67]

12. XRE family transcriptional regulator [Burkholderia sp. YI23] 
Ceara 02 sequences and putative proteins

1. hypothetical protein BMULJ_05097 [Burkholderia multivorans ATCC 17616]

2. ATPase [Burkholderia phytofirmans]

3. hypothetical protein OI25_7016 [Burkholderia fungorum]

4. ATPase [Burkholderia ginsengisoli]

5. ATPase [Burkholderia dilworthii]

6. ATPase [Burkholderia bryophila]

7. long-chain fatty acid--CoA ligase [Burkholderia sp. H160]

8. ATPase [Burkholderia caledonica]

\section{Copyrights}

Copyright for this article is retained by the author(s), with first publication rights granted to the journal.

This is an open-access article distributed under the terms and conditions of the Creative Commons Attribution license (http://creativecommons.org/licenses/by/3.0/). 